\title{
Estimating Coherent Optical Transmission System Performance over Heterogeneous Fibre Span Lengths
}

\author{
Hou-Man Chin*, David J. Ives ${ }^{+}$, Seb J. Savory ${ }^{+}$, Jarosław P. Turkiewicz* \\ *Orange Polska, Al. Jerozolimskie 160, 02-326 Warsaw, Poland \\ ${ }^{+}$University of Cambridge, 9 JJ Thomson Avenue, Cambridge, CB3-OFA, U.K \\ E-mail:Hou-Man.Chin@orange.com
}

\begin{abstract}
This work investigates the estimation accuracy of a coherent optical transmission system's performance when the number of spans in the system is known and the overall system length is approximately known. However, there is no knowledge of the lengths of the individual spans as may happen when institutional knowledge of deployed fibre is lost. Estimating the performance of an optical signal over systems using this fibre may be inaccurate as a result. In this paper, the estimation accuracy of this scenario is examined considering a system with heterogeneous fibre span lengths for a DWDM system transmitting 80 channels modulating polarization multiplexed quadrature phase shift keying (PM-QPSK) at 32 Gbaud across the optical C-band using the Gaussian noise (GN) model in comparison to a reference system with homogenous fibre spans.
\end{abstract}

Keywords: optical fibre communication, systems analysis and design

\section{INTRODUCTION}

The ever-increasing demand for bandwidth has increased exponentially over the past few decades. This demand is driven by the ever-increasing numbers of networked devices per person in addition to high bandwidth demand applications such as cloud computing and video streaming [1]. These are expected to increase global IP traffic almost three-fold in the period between 2015 and 2020. To service this demand, there are a few options, one is to deploy new optical networks, another is to deploy new transceivers [2] onto existing networks which have space for additional optical channels, the last is to upgrade existing transceivers to higher bandwidth ones. The last two options require knowledge of the optical network to properly plan deployments. It may be that the total system length is approximately known in addition to the number of spans, however the exact details of the fibre span lengths is unknown. The GN model [3] is a well-known model used to estimate the performance of coherent optical signals over dispersion uncompensated transmission systems. In this work, we use it to examine the impact on the estimation accuracy of coherent optical transmission systems with heterogenous span lengths.

\section{SYSTEM SETUP}

Figure 1 shows the coherent optical transmission DWDM system examined in this work. It is a 32 Gbaud system transmitting polarization multiplexed quadrature phase shift keying (PM-QPSK) on 80 channels spaced at $50 \mathrm{GHz}$ across the optical C-band as per ITU standard G.694.1 [4]. The channels propagate over 10 spans of standard single mode fibre (SMF) with the following properties: attenuation $\alpha=0.22 \mathrm{~dB} / \mathrm{km}, D=16.7 \mathrm{ps} /(\mathrm{nm}$. $\mathrm{km})$ with a nonlinear coefficient $\gamma=1.3 \mathrm{~W} / \mathrm{km}$. The system is assumed to have ideal root raised cosine pulse shaping and the back to back $\mathrm{SNR}_{0}$ is $20 \mathrm{~dB}$. The amplifier after each fibre span have noise figure $\mathrm{NF}=5 \mathrm{~dB}$ and operate such that the power into each span is the same as the transmitter launch power. The lengths of the fibre spans are randomly chosen from 40 to $120 \mathrm{~km}$, in $1 \mathrm{~km}$ granularity and with equal probability. A Monte Carlo simulation of 100,000

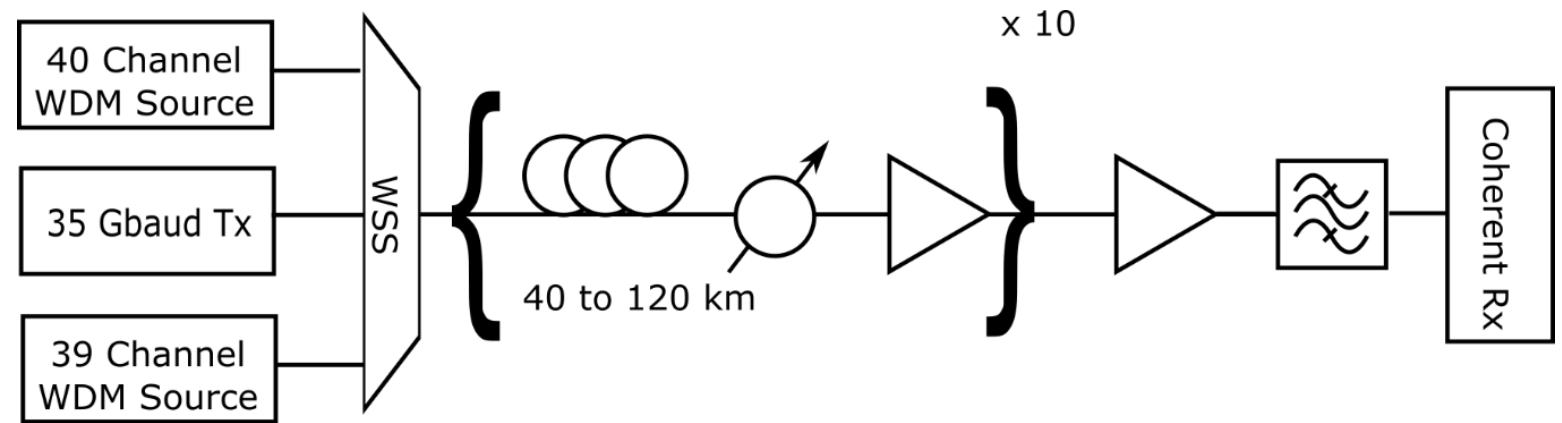

Figure 1. Transmission system setup under examination 


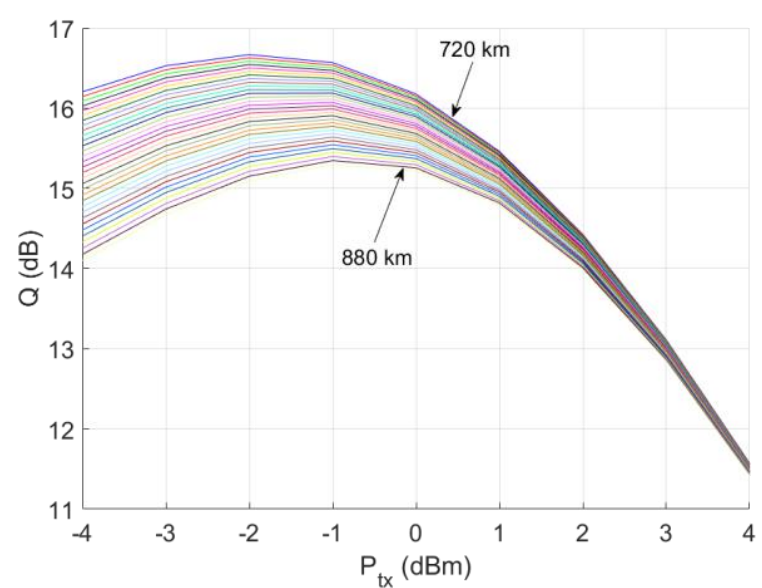

Figure 2. Reference $Q(\mathrm{~dB})$ performance for 10 spans of 72 to $88 \mathrm{~km}$ fibre

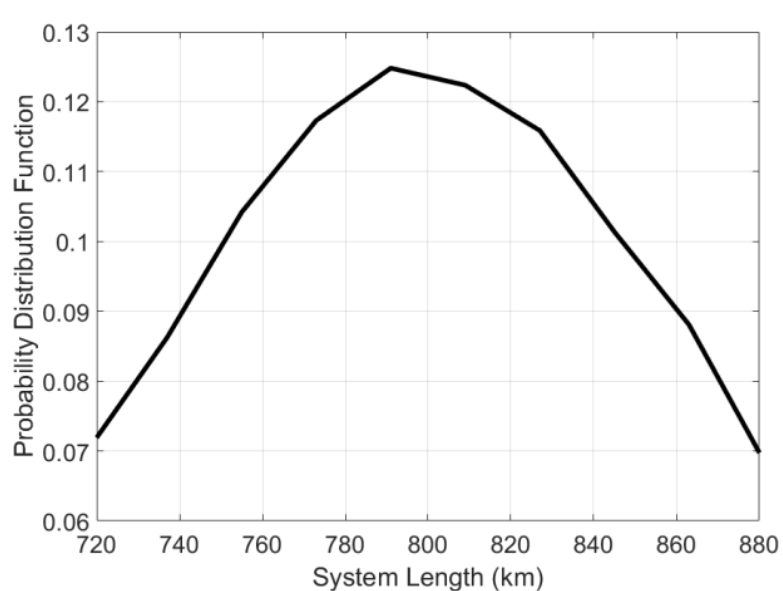

Figure 3. Probability distribution function of the total system length from the Monte Carlo simulation

of such span arrangements were performed, sweeping the transmitter power from -4 to $4 \mathrm{dBm}$ in $1 \mathrm{~dB}$ steps. The results with a total system length of $720 \mathrm{~km}$ to $880 \mathrm{~km}$ were examined, circa 72,000 in total. These total lengths were chosen such that they are within $10 \%$ of a typical $10 \times 80 \mathrm{~km}$ fibre span system length.

\section{Results}

Figure 2 shows the performance of the 10-span system with identical spans ranging from 72 to $88 \mathrm{~km}$ in length in Q where $Q=20 \times \log _{10}\left(\sqrt{2} \times \operatorname{erf} c^{-1}(B E R)\right)$ where BER is the bit error rate. Figure 3 shows the probability distribution function of the total system length arising from the random selection of fibre span length from 40 to $120 \mathrm{~km}$ with equal likelihood. To assess the inaccuracy arising from heterogenous span lengths mapping onto the total system length, the BER performance for systems with a total fibre length within a $5 \mathrm{~km}$ window are averaged and then converted to Q. This averaged performance is shown in Figure 4 and it can be clearly seen that the optimum launch power is approximately $0 \mathrm{dBm}$ on average. The systems with a shorter total length perform better than ones with longer length while system performance in the linear and nonlinear transmission regimes deteriorate from the optimum at $0 \mathrm{dBm}$ as expected.

Figure 5 shows the difference between the Q of the averaged BER performance for a total system length and the Q performance for a system with $10 \times 80 \mathrm{~km}$ length fibre spans. It can be immediately seen that the greatest error in estimation occurs when the transmission system is longer than the $800 \mathrm{~km}$ total length of the reference system with up to $3 \mathrm{~dB}$ in estimation error at $880 \mathrm{~km}$ total system length when transmitting at $-4 \mathrm{dBm}$. The minimum error of less than $0.5 \mathrm{~dB}$ occurs in the opposite regime of when the total system length is short, in this case equalling $720 \mathrm{~km}$ at $4 \mathrm{dBm}$. It should be noted that even for a total system length of $800 \mathrm{~km}$, equalling the reference system length there is still at least $0.5 \mathrm{~dB}$ error, at the previously determined optimum launch power, there is a $1 \mathrm{~dB}$ estimation error. This is because the $800 \mathrm{~km}$ systems compared to the reference, while having the same total length, have heterogeneous spans which add up to approximately $800 \mathrm{~km}$. This error is increased at lower launch powers

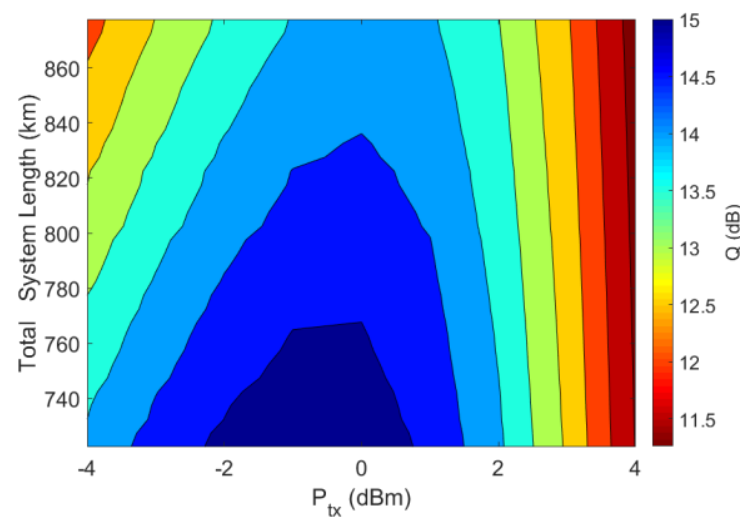

Figure 4. $Q$ performance with respect to transmitter launch power and total system length per $5 \mathrm{~km}$ bin

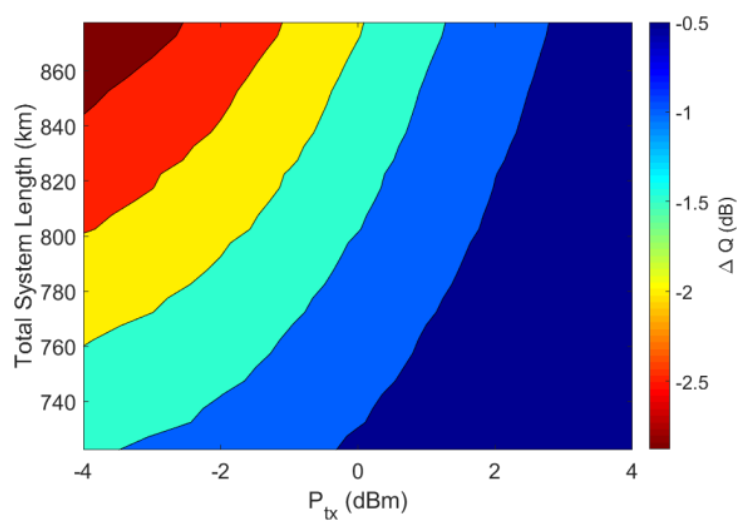

Figure 5. $Q$ calculated from averaged BER compared to a reference $10 \times 80 \mathrm{~km}$ system 


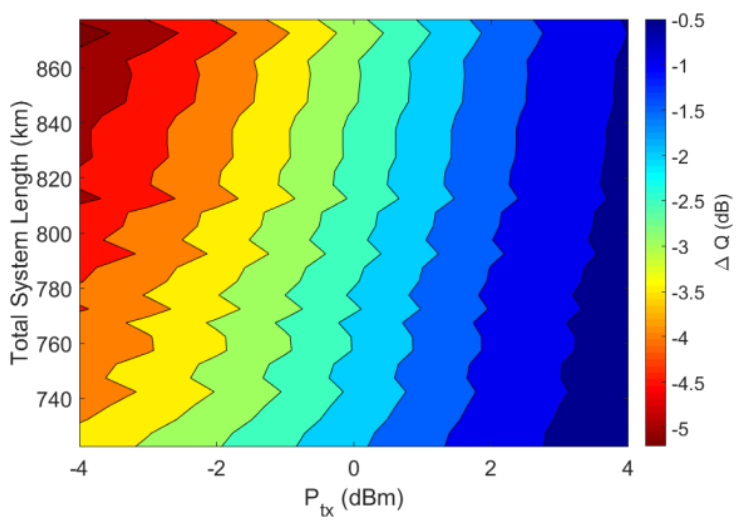

Figure 6. $Q$ calculated from the worst case BER compared to a reference $10 \times 80 \mathrm{~km}$ system

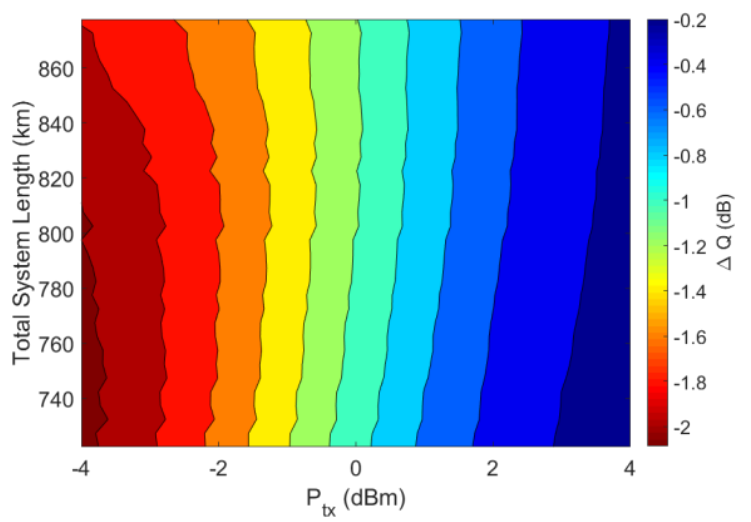

Figure 7. $Q$ calculated from averaged BER compared to a reference system of the same total length

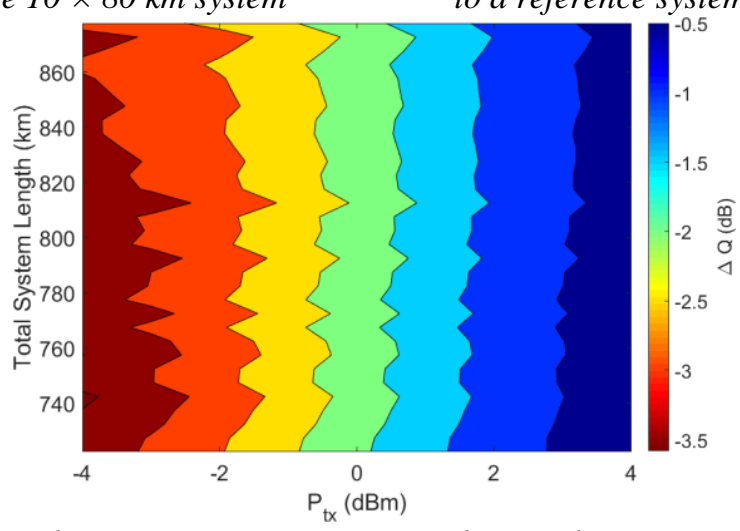

Figure 8. Q calculated from the worst case BER compared to a reference system of the same total length

up to $2 \mathrm{~dB}$. Figure 6 shows the comparison with the worst performing span arrangement, the maximum error increases to $\sim 5 \mathrm{~dB}$ while the minimum remains at $0.5 \mathrm{~dB}$, occurring for a long transmission system operating at low power and a short system operating at high power respectively, comparing the worst case $\sim 800 \mathrm{~km}$ system to the reference system there is $\sim 2.5 \mathrm{~dB}$ estimation error. The jaggedness is the contours is due to the different total system lengths equating to different span arrangements. The averaged performance of systems longer than the 800 $\mathrm{km}$ reference system was up to $1 \mathrm{~dB}$ additional error over those shorter than $800 \mathrm{~km}$.

Figure 7 shows the same Q of the averaged BER performance from Figure 4 but in comparison to a reference system consisting of 10 spans of identical length, i.e. the $720 \mathrm{~km}$ total length system is $10 \times 72 \mathrm{~km}$ spans and the $840 \mathrm{~km}$ reference system is $10 \times 84 \mathrm{~km}$ fibre spans. It also shows that the maximum error has decreased from $\sim 3$ $\mathrm{dB}$ to $\sim 2 \mathrm{~dB}$ while the minimum error has also decreased from $\sim 0.5 \mathrm{~dB}$ to $\sim 0.2 \mathrm{~dB}$. The regions where these occur have however changed. The maximum error occurs at shorter total system lengths at the lowest optical launch power used here $\mathrm{P}_{\mathrm{tx}}=-4 \mathrm{dBm}$. The minimum error does occur at the same location of a short system with high launch power $\mathrm{P}_{\mathrm{tx}}=4 \mathrm{dBm}$. The estimation error is now relatively consistent with respect to transmitter launch power, with $\sim 0.2 \mathrm{~dB}$ differential between the shortest and longest systems examined, Figure 5 shows this error to be up to $\sim 1.5 \mathrm{~dB}$. Hence using the total system length is a better overall estimate than assuming a standard span length and then using the number of spans to calculate the total system length. It must be noted that for the composite systems' optimum launch power from Figure 4, the average estimation error remains approximately 1 $\mathrm{dB}$ regardless of total system length. Figure 8 shows the comparison with respect to the worst case for the same reference systems as in Figure 7. The maximum estimation error is $3.5 \mathrm{~dB}$ and the minimum is $0.5 \mathrm{~dB}$. Like in Figure 7, the most and least accurate estimation are for short length systems at low and high power respectively. At the optimum launch power for averaged system performance, there is $\sim 2 \mathrm{~dB}$ estimation error in the worst case.

\section{CONCLUSIONS}

This work investigated the error that can occur in estimating the performance of coherent optical transmission systems with heterogeneous span lengths over systems with a total number of 10 fibre spans. The investigation was performed with Monte Carlo simulations of 100,000 permutations of span arrangements with span lengths randomly selected with equal likelihood from 40 to $120 \mathrm{~km}$ lengths. Each arrangement was evaluated for a transmitter optical launch power ranging from -4 to $4 \mathrm{dBm}$. The systems with a total fibre length ranging from 720 $\mathrm{km}$ to $880 \mathrm{~km}$ were selected for further examination. The estimated performance are grouped together according to their respective total system length in $5 \mathrm{~km}$ bins. Each bin's system BER performances were averaged. This 
average was first compared to the performance of a reference $10 \times 80 \mathrm{~km}$ span system found that the estimation error ranged from 0.5 to $3 \mathrm{~dB}(\mathrm{Q})$. Notably, for $\sim 800 \mathrm{~km}$ systems, the estimation was on average $\sim 1 \mathrm{~dB}$ in error at the optimum launch power because while the total system length may be approximately the same, the lengths of the fibre spans the system consists of is unlikely to be all $80 \mathrm{~km}$. In the worst case, the error ranged from 0.5 to 5 $\mathrm{dB}(\mathrm{Q})$. The averaged performance estimations were then compared to systems of equivalent total system length but with homogenous span lengths. It was found that the error in estimation decreased to a 0.2 to $2 \mathrm{~dB}(\mathrm{Q})$ range. The estimation error at the optimum launch power did not decease and remained $\sim 1 \mathrm{~dB}(\mathrm{Q})$, regardless of system length but the error decreased with increasing optical launch power and vice versa. Comparing with the worst performing span arrangement gave 0.5 to $3.5 \mathrm{~dB}(\mathrm{Q})$ in estimation error and the estimation error at the optimum launch power is $\sim 2 \mathrm{~dB}(\mathrm{Q})$, regardless of total system length.

In summary, the performance estimation of a system with heterogeneous span lengths must be done with caution since large errors may occur. Any error in the performance estimation during the design stage would have to be potentially absorbed by the provisioned system margin, leading to the requirement to provision extra margin which may impact system capacity.

\section{ACKNOWLEDGEMENTS}

The authors acknowledge support by EU ICONE project grant \#608099.

\section{REFERENCES}

[1] Cisco, “Cisco VNI Forecast and Methodology, 2015-2020,” Tech. rep., Cisco (2016).

[2] Kim Roberts, Qunbi Zhuge, Inder Monga, Sebastien Gareau, and Charles Laperle, "Beyond 100 Gb/s: Capacity, Flexibility, and Network Optimization [Invited]," in J. Opt. Commun. Netw. 9, C12-C24, (2017)

[3] Poggiolini, P., "The GN Model of Non-Linear Propagation in Uncompensated Coherent Optical Systems," in J. of Lightw. Tech., 30(24), 3857-3879 (2012).

[4] "Spectral grids for WDM applications: DWDM frequency grid", G.694.1, ITU-T Std. 